\title{
Measurement of gamma-rays from neutron-oxygen reaction for neutrino-nucleus interaction
}

\author{
T.Horai ${ }^{* 1}$ for the E525 Collaboration* \\ ${ }^{1}$ Okayama university, Okayama-shi, Okayama, 7008530, Japan \\ E-mail: 551horaies.okayama-u.ac.jp
}

\begin{abstract}
Neutrino neutral-current elastic interaction by atmospheric neutrinos is one of the main background in Supernova relic neutrino (SRN) search at Super-Kamiokande with Gadolinium. Understanding of gamma-ray production via neutron interaction on oxygen is important for the study of neutrino neutral-current elastic interactions. A measurement of gamma-rays production from such reactions was performed by using a $30 \mathrm{MeV}$ quasi-mono energetic neutron beam in the Research Center for Nuclear Physics, Osaka University. In this proceedings the current status and future prospects of this mesurement are presented.
\end{abstract}

The 4th KMI International Symposium (KMI2019)

18-20, February 2019

Nagoya, Japan

${ }^{*}$ Speaker. 


\section{Introduction}

The atmospheric neutrino neutral-current (NC) elastic scattering is one of the main background in supernova relic neutrino (SRN) search. This background cannot be distinguished from SRN in Super-Kamiokande (SK), therefore it should be estimated by a simulation. NC elastic interaction cross section is measured by the T2K long baseline neutrino experiment [1,2]. In this experiment, the neutrino peak energy $(\sim 600 \mathrm{MeV})$ is near the peak of the atmospheric neutrino flux. However, the result suffers from large systematic errors that is caused by nucleons emitted by neutrinooxygen interaction. Especially, neutrons interact with other oxygen inside SK leading to additional gamma-rays (secondary gamma-rays : Figure.1). Secondary gamma-rays are difficult to distinguish from primary neutrino-oxygen gamma-rays because they have similar energies and are emitted within $\mathrm{O}(10)$ ns. Neutron beam experiment (E487) was carried out in Research Center for Nuclear Physics (RCNP) in Osaka University [4-6] for the understanding of secondary gamma-rays from neutron-oxygen interactions [3] to provide data that can help understanding of secondary gammarays. Similar experiment, E525, is conducted with different neutron energy. Neutron flux, emitted by the $\mathrm{T} 2 \mathrm{~K}$ experiment neutrino-oxygen $\mathrm{NC}$ reactions, is estimated and $30 \mathrm{MeV}$ is the peak energy and falls gradually to $300 \mathrm{MeV}$. E487 experiment used $80 \mathrm{MeV}$ and E525 used 30 and $250 \mathrm{MeV}$ for further verification. This proceedings reports current status from the E525 experiment.

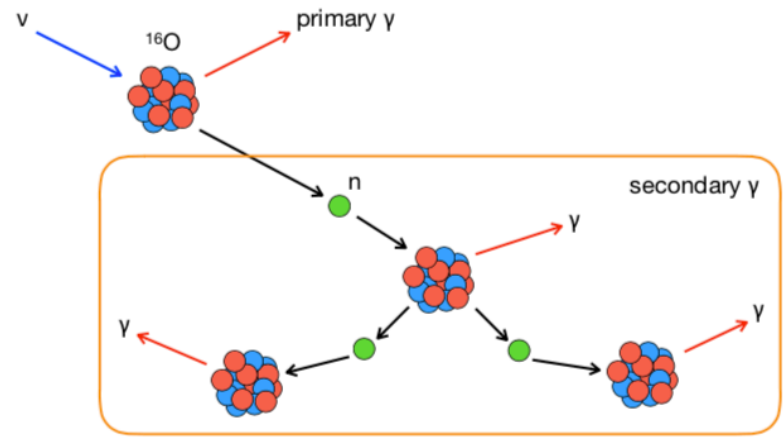

Figure 1: Process of primary and secondary gamma-ray production in water. (This picture taken from Ref.[3])

\section{E525 Experiment}

E525 experiment was carried out at the RCNP in October and December, 2018. A proton beam is accelerated by two cyclotrons and interact with lithium target to produce an almost mono energetic neutron beam via the ${ }^{7} \mathrm{Li}(\mathrm{p}, \mathrm{n})^{7} \mathrm{Be}$ reaction. Mono energetic neutron can decrease contamination from neutron of other energy. The energies of proton are $30 \mathrm{MeV}$ and $250 \mathrm{MeV}$.

A cylindrical acrylic container was placed on the beam axis, and container was filled with water or air. A high-purity germanium (HPGe) detector was placed upstream of the water target to measure gamma-rays. The HPGe was read out by a USB-MCA APG7300L and a 14-bit CAEN DT5725 Flash-ADC.

Neutron beam flux and the backgrounds from neutrons scattered in the water-filled target ware 
also measured. For the neutron beam flux measurement, the water target was replaced with an organic liquid scintillator (BC-501A, Saint-Gobain 20LA32). The BC-501A coupled to Hamamatsu H6527 PMT was read out by the Flash-ADC. A CsI(Tl) crystal coupled to the H6410 PMT was also placed upstream of the water target and measured scattered neutrons.

For the normalization, the proton beam current was monitored by the Faraday cup during the experiment.

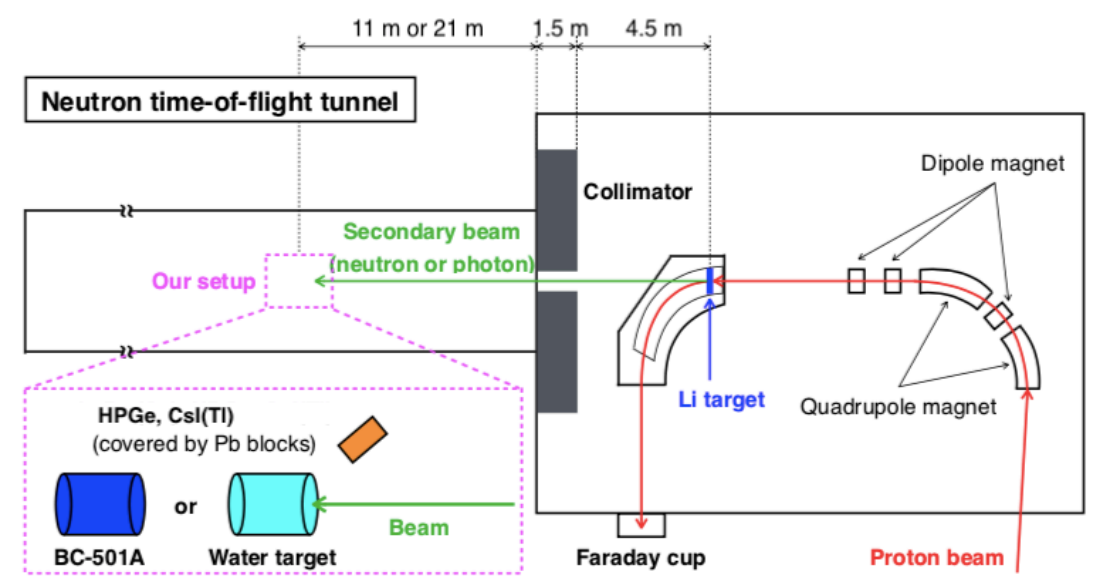

Figure 2: Schematic view of the E525 experiment.

\section{Analysis of the $30 \mathrm{MeV}$ experiment}

\subsection{Gamma-ray Spectrum}

Figure 3 shows energy spectrum of the HPGe. Several peaks similar to the previous experiment were observed. The $6.13 \mathrm{MeV}$ gamma-ray was produced from inelastic scattering on ${ }^{16} \mathrm{O}$. The 5.27 $\mathrm{MeV}$ gamma-ray was produced from nucleon knock-out flipping a deuteron, or nuclear decay after elastic scattering. The $4.44 \mathrm{MeV}$ gamma-ray was produced from alpha knock-out or the decay of ${ }^{16} \mathrm{O}$ with an alpha emission. The physics processes that produce these gamma-rays are summarized in Table 1. See Ref.[3] for more details of the physics processes.

\begin{tabular}{cc}
\hline \hline Energy[MeV] & Physics process \\
\hline 6.13 & ${ }^{16} \mathrm{O}\left(\mathrm{n}, \mathrm{n}^{\prime}\right)^{16} \mathrm{O}^{*}$ \\
5.27 & ${ }^{16} \mathrm{O}\left(\mathrm{n}, \mathrm{n}^{\prime}\right)^{16} \mathrm{O}^{*}$ then ${ }^{16} \mathrm{O}^{*} \rightarrow{ }^{15} \mathrm{~N}^{*}+\mathrm{p}$, or ${ }^{16} \mathrm{O}(\mathrm{n}, \mathrm{np})^{15} \mathrm{~N}^{*}$, or ${ }^{16} \mathrm{O}(\mathrm{n}, \mathrm{d})^{15} \mathrm{~N}^{*}$ \\
4.44 & ${ }^{16} \mathrm{O}\left(\mathrm{n}, \mathrm{n}^{\prime}\right)^{16} \mathrm{O}^{*}$ then ${ }^{16} \mathrm{O}^{*} \rightarrow{ }^{12} \mathrm{C}^{*}+\alpha$, or ${ }^{16} \mathrm{O}(\mathrm{n}, \mathrm{n} \alpha){ }^{12} \mathrm{C}^{*}$ \\
\hline \hline
\end{tabular}

Table 1: Observed gamma-rays from neutron- ${ }^{16} \mathrm{O}$ interaction with their parent processes.

\subsection{Neutron Flux}

For the gamma-ray cross section analysis, neutron flux was estimated by BC-501A data. First, neutron-like events are selected using Pulse Shape Discrimination (PSD) method. Second, kinetic 


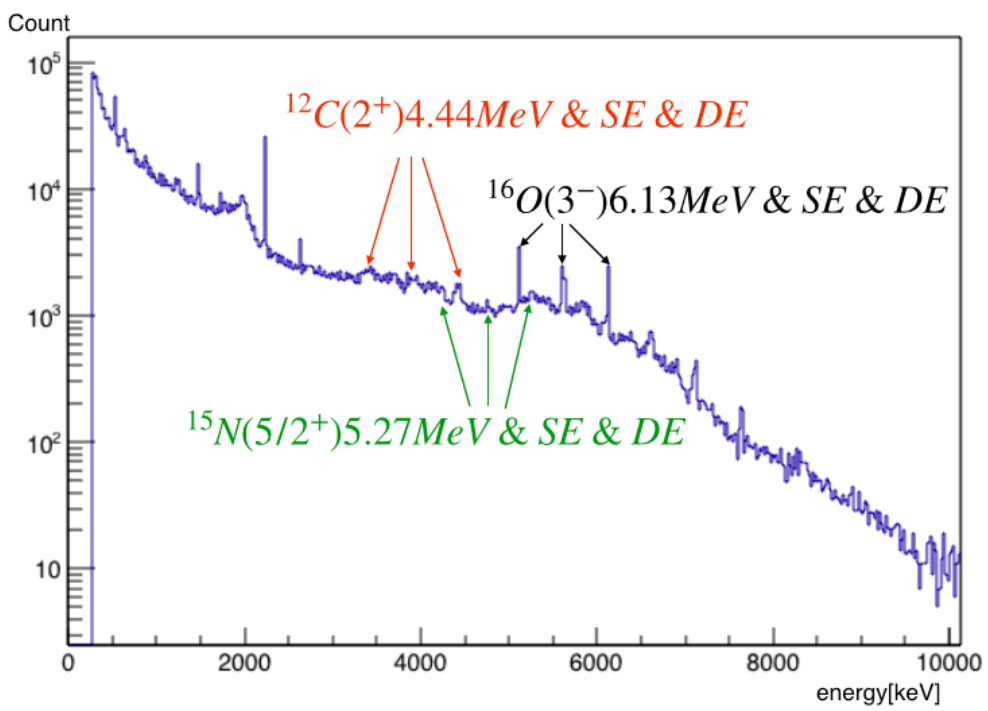

Figure 3: Energy spectrum of the HPGe in $30 \mathrm{MeV}$ experiment.

energy is reconstructed from their TOF information. Finally, The neutron flux is obtained from the kinetic energy distribution normalized by detection efficiency, detector acceptance, and proton beam current. Detection efficiency is calculated by using the SCINFUL-QMD [7, 8] simulation and the result is shown in Figure 4.

Figure 5 shows the neutron beam flux. Peak region is 16 to $26 \mathrm{MeV}$ and only this region is used in neutron cross section measurement. In order to reduce neutron background, a beam profile was measured before gamma-ray measurements. BC501-A was shifted from the beam axis to 24 $\mathrm{cm}$ perpendicularly off-axis in steps $6 \mathrm{~cm}$. Figure 6 shows beam profile, the neutron flux at $24 \mathrm{~cm}$ away from beam center is two orders smaller than center one so that the gamma-ray detector placed at this point to reduce neutron background. Beam stability was monitored during the beam test, as shown in Figure 7. It has still uncertainty, and the analysis to reduce it is now on going.
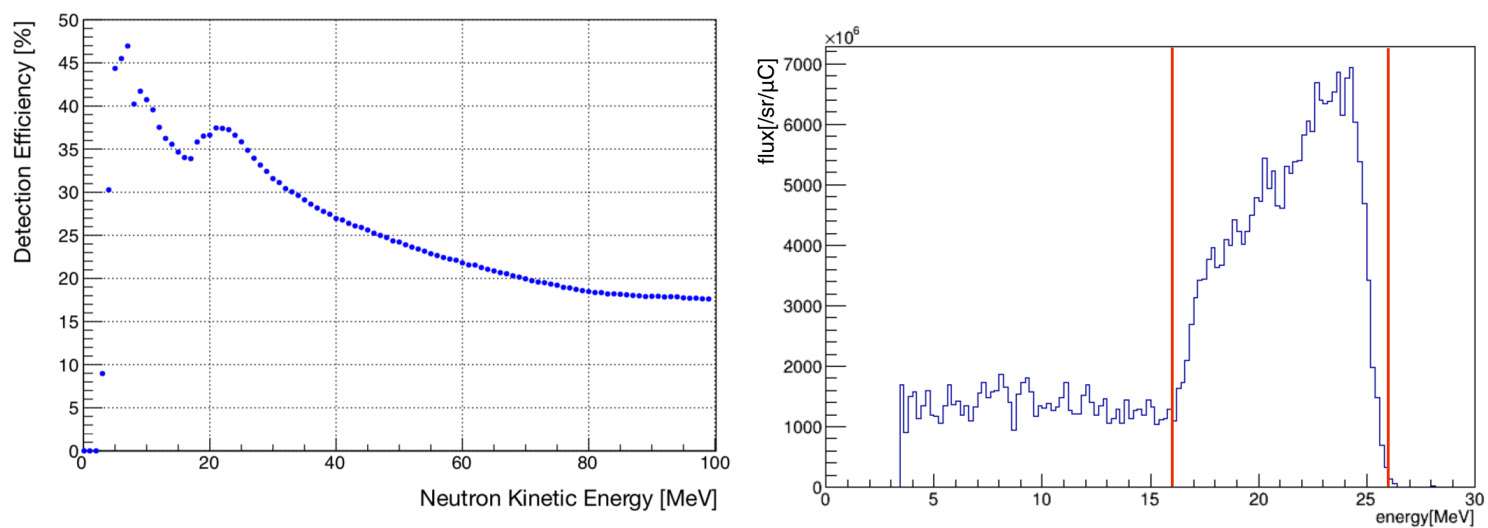

Figure 4: Neutron detection efficiency calculated by SCINFUL-QMD.

Figure 5: Neutron beam flux in $30 \mathrm{MeV}$ experiment. 

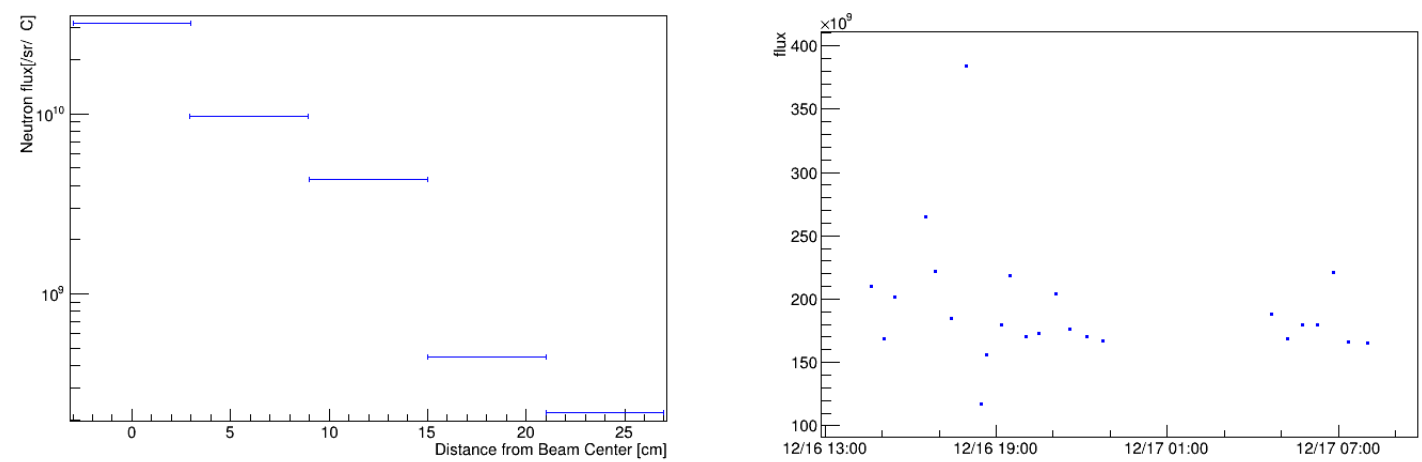

Figure 6: Neutron beam profile in $30 \mathrm{MeV}$ experiment.

Figure 7: Beam stability during the experiment.

\section{Status and Conclusion}

At present, the E525 experimental analysis is in progress. In the $30 \mathrm{MeV}$ case, neutron beam peak region is 16 to $26 \mathrm{MeV}$, and this range will be used for analysis . From now on, systematic uncertainty of neutron flux should be estimated and gamma-ray cross section analysis will start.

\section{Acknowledgment}

We are grateful to the RCNP staff for giving a beam time and accelerator group. This work was supported by JSPS KAKENHI Grant Number JP26400292.

\section{References}

[1] K. Abe et al. (T2K Collaboration), Nucl. Instr. and Meth. Phys. Res. A, 659 (2011).

[2] K. Abe et al: (T2K Collaboration), Phys. Rev. D 90, 072012 (2014).

[3] Y. Ashida et al., arXiv:1902.08964 [nucl-ex] (2019).

[4] T. Miura et al., Proceedings of 13th International Conference on Cyclotrons and their Applications, Vancouver, Canada (1992).

[5] T. Saito et al., Proceedings of 14th International Conference on Cyclotrons and their Applications, Cape Town, South Africa (1995).

[6] S. Ninomiya et al., Proceedings of 17th International Conference on Cyclotrons and their Applications, Tokyo, Japan (2004).

[7] D. Satoh et al., JAEA-DATA/CODE 2006-023 (2006).

[8] T. Kajimoto et al., Nucl. Instr. and Meth. Phys. Res. A 665 (2011). 